\title{
Effective Counseling Methods to Overcome the Psychological Factor of a Sportsperson
}

\author{
C. Veerender \\ Psychologist, You \& Me, counselling \& P.D. Training Center, \\ Hyderabad, Telangana State, India. \\ drveerender@gmail.com
}

\begin{abstract}
--performance in sorts is not just a physiology and biochemical by-product, but psychological factors also play a crucial role in outcome goal of a player. However, every player has a certain stress level that is needed to optimize his or her performance in a game, and that depends on factors such as past experiences, practices, learning the techniques, coping responses, and genetics. A Player that is high anxious is expected to play less well, and experience great shame, upset, and more frequent criticism from parents and coaches in the event of poor performance. Even in the absent of these expectancies, high-anxious players worries more frequently than low-anxious players about not playing well, losing, and being evaluated by parents, coaches, and teammates. According to Arnold Palmer a professional golfer, every game is $\mathbf{9 0 \%}$ psychological. Stress result from nonfulfilment of needs, and continuous stress creates anxiety; and anxiety leads to tension. Stress can ruin a sportsperson's performance, and it can enhance his/her performance; depending on how the individual takes it. Physiological factors that influence the performance of players include stress, anxiety, tension, and aggression. Counselling of sportsmen or players is not just to make them comfortable with stress and anxiety, but also to help them overcome their low self-esteem and gain a better self-esteem; and to help them develop positive belief on their learned skills and techniques, so they can perform better in competitions. This paper addresses the hampering performance of players, and tools to handle them to enhance their performance.
\end{abstract}

Keywords—counseling, psychological, sportsperson

\section{INTRODUCTION}

Every sportsperson always face a challenging situation in national and international competitions, irrespective of their practice sessions, having great coach supervision, successful player's suggestions, having great belief in self to perform well in the competition. Still, due to the stress, results are never the same as expected by the players and coaches; therefore, new question arises that "what we can do so that the players give their best in spite of the stress?" In this scenario, that is where the psychologist service appears to help the players ease out their stress, so they can perform better. In addition to training the body, the brain that is the top portion of the body where training is deposited, and energies are channelled needs extra training to perform well in the field. In the past, least priority was given to train the brain except for few professionals, which takes the help of sports psychologists. Slowly, the coaches and the players have started realizing the role of counselling in enhancing players performance not only to recover after injury, but also in regular performances.
Stress results from non-fulfilment of needs, and continuous stress creates anxiety, which leads to tension. Tension can also break focus thereby leading to failure. Psychological factors that influence the performance of players are stress, anxiety, tension, and aggression. This paper addresses the issue hampering the performance of a player, and tools to handle them to enhance their performance.

\section{PSYCHOLOGICAL FACTORS INFLUENCING PLAYER'S PERFORMANCE}

A. Stress

Every competitive sports person experiences some stress; either good or bad. Stress may positive and helpful or an instil anxiety and apprehension. Also, pregame jitters can cause some athletes sleeplessness before the competition while some players may not be able to eat anything before a big game, This is because they feel excitement or thrill in anticipation for the competition (what I can call positive pregame jitters). A high level of activation help players to perform their best up to a point where they may be too jacked to play well Also, too little or too much intensity or stress can cause some players performance to decline. Ability to cope effectively with pregame nerves is crucial to consistent peak performance. See figure 1(the stress response curve).

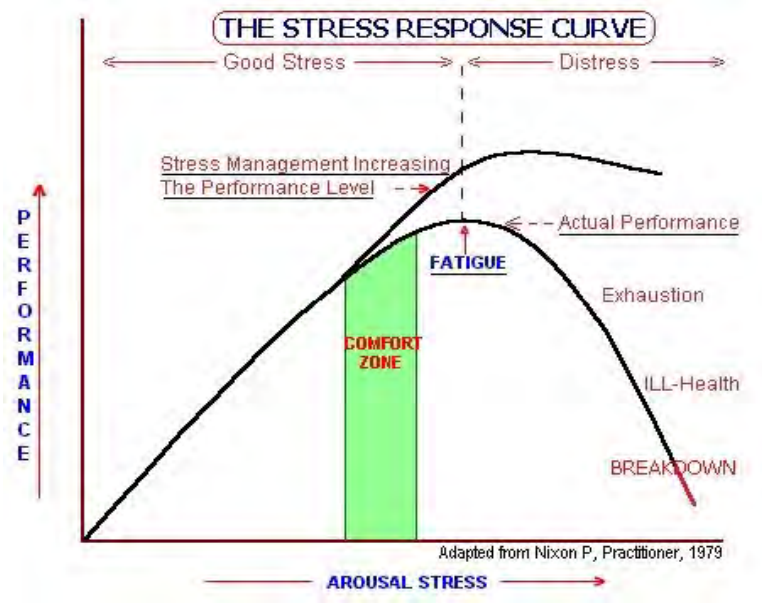

Fig. 1. The stress performance curve

Nixon, P. (1979), created the stress performance curve above and it explains how stress affects performance in theoretical terms. The Stress Response Curve shows that as stress level increases, the performance level also increases to the point of eustress, or healthy tension. Near the point of fatigue, there is an identified area called the Comfort Zone, which indicates the range of stress level that can be absolutely managed, that 
facilitates good performance levels. Once stress begins to be perceived as overwhelming or excessive, an individual reaches a fatigue point where the performance level starts to decline. The ultimate end of overwhelming stress, called burnout, can be exhaustion, ill health or breakdown.

\section{B. Anxiety}

The medical definition of anxiety describes anxiety as a state that consist of physiological and physical symptoms, brought about by a sense of apprehension of a perceived threat. Anxiety trait relates to an aspect of personality in which nervousness is a stable trait in an individual.

Anxiety in performance in sport sometimes referred to as 'choking,' is described as a decrease in athletic performance due to too much-perceived stress. There seems to be increase in an athlete perceived stress because they have an audience, and they have an extremely high expectation of their success. This type of stress is often based on the way the athletes' interprets the situation. It is rarely an external situation that causes stress, but rather the way the athlete's self-talk describes the situation that creates feelings of stress, anxiety, and fear.

For athletes who choke during competitions, it is important to understand that the thoughts they have regarding the event can be modified, adjusted or controlled with appropriate sports psychology and mental practice. Players should determine first if their thoughts of doubt, failure or a lack of confidence is due to a perceived lack of ability. If so, self-talking will generally lead to continuous feeling of anxiety, nervousness, and tension. Players need to realize that it is tough to do your best in a sport when your own internal voice is telling you otherwise. To overcome performance anxiety, a sports psychologist, coach, and trainer may try to help the athlete understand why those thoughts and feelings develops, and they try to change or modify that process with limited amounts of success. Players returning from injuries often have emotional issues that can undermine their confidence. Such thoughts may arise be of interest, but knowing the answer is not always necessary to overcome them.

\section{Tension}

Tension is the state of body and mind, which results from internal and/or forces acting in opposition to each other such as in emotional reactions like anger or fear. In one sense, tension may refer to the residual effect of mental or emotional strain reflected in a person's appearance and/or behaviour; and in another sense, it means tonus-a state of partial contraction between muscles that are not actively working. Excessive tonus is also called tension, which may result, from excessive stimulation, or from strains and stress on an individual. This kind of tension is not as harmful as the one that arises from a state of persistent unsatisfied wants and desires. Physiologically and psychologically, as long as an individual wants are unsatisfied, he/she remains in a state of tension.

\section{Aggression}

Aggression is any kind of behaviour that is carried out with the intention of harming another person. Baron (1977) addressed this issue in his influential distinction between hostile and instrumental aggression. Hostile aggression takes place when the primary intention of the behaviour is to harm other players. This type of aggression is accompanied by anger and the underlying wish is to see the victim suffer. Instrumental aggression take place when the behaviour is clearly likely to cause harm, but the intention is to achieve a different aim; such as to score a point or prevent the opposition from scoring a goal. Husman \& Silva (1984) made further distinction between aggression and assertiveness. According to Husman \& Silva (1984), assertiveness involves the type of behaviour that might appear aggressive, but does not result in harming an opponent. In many sports for example, one might choose at certain times to charge directly towards an opponent, perhaps with an accompanying cry, but without any intention of making contact.

\section{METHODS TO OVERCOME FROM THE ABOVE FACTORS TO ENHANCE THE PERFORMANCE OF A PLAYER}

\section{A. Relaxation}

Some athletes suffer from low level of arousal, while some suffer from excessively high level of anxiety and tension, which creates problems that are more difficult. For these athletes, any strategy calculated to increase arousal can only cause grater anxiety and tension. Relaxation procedures can be adequately categorized under the broad heading of relaxation. These include:

1. Progressive relaxation

2. Autogenic training

3. Meditation

4. Biofeedback

Each procedure is unique, but they all yield essentially the same physiological result i.e. they all result in the same relaxation response. Such procedure results in decreased oxygen consumption, heart rate, respiration, and skeletal muscles activity, while they increase skin resistance and brain waves.

Four different factors are necessary for eliciting relaxation response. These are a mental device, a passive attitude, decreased muscle tone, and a quiet environment. Each of these factors is present to some degree in specific relaxation technique that will be discussed. The mental device is generally some sort of word, phrase, object or process used in shifting attention in wards.

\section{B. Progressive relaxation}

Jacobson's relaxation technique is a type of therapy that focuses on tightening and relaxing specific muscle groups in sequence. It is also known as progressive relaxation therapy. By concentrating on specific areas, tensing them and then relaxing them, you can become more aware of your body and physical sensations. Edmund Jacobson invented this technique in the 1920 s as a way to help his patients deal with anxiety. Dr. Jacobson felt that relaxing the muscles could relax the mind as well. Therefore, the technique involves tightening one muscle group while keeping the rest of the body relaxed, and then releasing the tension. 


\section{Autogenic Training}

Autogenic training relies on feelings associated with the limbs and muscles of the body. Autogenic training is very similar to autohypnosis, and is based on early research with hypnosis. Various authors have suggested that different exercises and self-statements can help bring about relaxation response using autogenic training.

\section{Meditation}

Meditation, as a form of relaxation, is tied directly to the concept of selective attention. In practicing meditation, the individual attempts to focus his/her attention on a single thought, sound, or object. The practice of meditation as a form of relaxation and thought control has its origin in eastern cultures more than four thousands of years ago. The most common mental device used in transcendental $\mathrm{m}$ editation is silent repetition of a mantra. It is unclear that the various forms of meditation can reduce anxiety and tension by evoking the relaxation response.

\section{E. Biofeedback Training}

It has been demonstrated that humans can voluntarily control functions of their autonomic nervous systems. Biofeedback is relatively a modern technique that is based on this principle. Biofeedback train ing instruments uses this to help people control their autonomic nervous systems. For example, a subject monitors can auditory a signal of her own heart rate and experiments with different thoughts, feelings, and sensations to slow the heart rate. Once the subject learns to recognize the feelings associate $\mathrm{d}$ with the reduction of heart rate, the instrument will be removed and the subject tries to control the heart rate without the use of the instrument.

\section{COGNitive Behaviour THERAPY (CBT)}

CBT is used to increase the unity of the observing and the participating self by challenging negative self-talk and providing more balanced reality-tested alternatives. Improved concentration on set goals such as improved motivation to win, improved self-control and (or) selfdiscipline, improved self-esteem and self-belief, improved self-understanding and self-awareness, improved timemanagement, and improved stamina in training. Majority of players have experienced loosing game in crucial competitions, this will then register in their sub conscious that some of the matches may not be successful. Their training will be good likewise; their learning technique, but their performance situation will be very disappointing. This kind of situations can easily be handled with the help of CBT. The basic idea of CBT stems from the thought and feelings, because they always go together; behind every bad feeling there is bad thinking. CBT can help handle this negative thought process with help of CBT structured process that can change the thinking pattern, and lead to realistic and practical thought process. This can make any sports person to perform better.

\section{NeURO-Linguistic PROGRAMMING}

NLP stands for Neuro-Linguistic Programming. Neuro refers to your neurology; linguistic refers to language; while programming refers to how that neural language functions. In other words, learning NLP is like learning the language of your own mind! Neuro-Linguistic Programming is like a user's manual for the brain, and taking NLP training is like learning how to become fluent in the language of your mind, so that the ever-so-helpful "server" i.e. your unconscious will finally understand what you actually want out of life. NLP is a study of the way in which human beings structure their perceptions, so that it can create a framework that can be used to analyse, study, reprogram, and program a person's behaviour, lifestyle, and attitude. Body posture, breathing, gestures towards the eyes, ears, body, eye movements, and language patterns are all elements that are used to trigger the unconscious mind in NLP. High-achievers and peak performers think, act, and feel differently from average people. They have superior mental strategies in place that help them learn faster and better to perform to their potential more often. Richard Bandler and John Grinder created Neuro-Linguistic Programming (NLP) in the 1970's.

NLP sports psychology helps to influence the human mind in such a way that it manages the internal state of the mind. The state of mind of an individualist an essential factor in bringing out his or her best performance. Many sports performers spend a lot of their time focusing on improving their fitness and technical ability, and often the mental element is neglected, even at the excellent level. Invoking state of mind can help bring out the best in a player. Coaches generally know their players very well, so they have an idea about the best thing to bring out the peak performance in a player.

\section{RATIONAL EMOTIVE BEHAVIOUR THERAPY'S (REBT)}

The central premise is that events alone do not cause a person to feel depressed, enraged, or highly anxious; rather, it is one's beliefs about the events, which contribute to unhealthy feelings and self-defeating behaviours. Rational Emotive Behaviour Therapy teaches the client to identify, evaluate, dispute, and act against his or her irrational self-defeating beliefs; thus helping the client to not only feel better but also get better. Rational Emotive Behaviour Therapy is an active-directive, solutionoriented therapy that focuses on resolving emotional, cognitive, and behavioural problems in clients. In other words, human beings based on their belief system actively, though not always consciously, disturb themselves about their disturbances. The Rational Emotive Behaviour Therapy framework assumes that humans have both rational and irrational tendencies. Irrational thought or images prevent goal attainment, leads to inner conflict, leads to more conflict with others and poor mental health. Rational thought or images lead to goal attainment and more inner harmony. In other words, rational beliefs reduce conflicts with others and improve health. 


\section{REFERENCE}

[1] H. N. Douglas, "Measurement And Evaluation In Physical Education \& Exercise Science", US, Gorsuch Scarisbric Publishers, 1989, p. 575 .

[2] S. J. Margaret, "Introduction to Measurement in Physical Education and Exercise Science", Toronto, Times Mirror/ Mosbt College Publishing, 1990, p. 566.

[3] K. K. Devinder, "Textbook of Applied Measurement Evaluation and Sports Selection". Delhi, Sports and Spiritual Science Publication, 2008, p. 530.
[4] H. M. Brrow, M. C. Rosmary, Gee, "Practical Approach to Measurement in Physical Education”, Philiadelohia:, Lea and Febiger, 1979.

[5] K. K. Suresh Kutty, "Guide for U. G. C. Examination for Physical Education", New Delhi, Sports Publication, 2004, p. 612.

[6] K. K. Suresh, "Foundation of Sports and Exercise Psychology", New Delhi, Sports Publications, 2004, p. 130.

[7] A. Singh, "Essentials of Physical Education $3^{\text {rd }}$ Edition", New Delhi, Kalyani Publisher's Ludhiana, 2008, p. 608

[8] M. L. Kamlesh, "Psychology of Physical Education and Sports", London, Boston Routleoge and Kagan Paul, 1972.

[9] C.Veerender, Proceedings of ICSM, 2017, pp569-577. 